\title{
Design and Implementation of Phased Array Antenna using Advanced Design System
}

\author{
Niranjan Ravi ${ }^{1}$, Vishal Sundarrajan ${ }^{2}$, Vetrivel DSB ${ }^{3}$ \\ BE, Department of Electronics and Communication, PSNACET, Dindigul, India ${ }^{1,2,3}$
}

\begin{abstract}
Phased arrays are a form of steerable antenna whose performance motivates the development of alternative structures which allow us to obtain simplified designs as powerful as the original approach but significantly cheaper. A System based design of phased array antenna using Advanced Design System is employed in ISM (The Industrial, Scientific and Medical) frequency range. Array antennas are used extensively in remote sensing applications, where a highly directive beam is needed to scan a particular area of interest on the surface of the earth. The study focuses on the design of different microstrip patch antenna arrays to be used in environmental sensing applications in the ISM frequency bands. Arrays of microstrip patches are easily printed on substrates using photolithography techniques and can be mass produced at cheap costs. In this paper we have implemented two types of array model using rectangular patch. Specifically, $1 \mathrm{X} 2$ and $2 \mathrm{X} 2$. This study includes design of feedback network, impedance matching, and return loss calculation. The substrate used is FR4 with dielectric constant of 4.6 and thickness about $1.6 \mathrm{~mm}$. The feed network is designed and optimized using ADS 2013 and then integrated with the array.
\end{abstract}

Keywords: Antenna, Array, Return Loss, Spacing, ADS (Advanced Design System)

\section{INTRODUCTION}

In antenna theory, a phased array is an array of antennas in which the relative phases of the respective signals feeding the antennas are varied in such a way that the effective radiation pattern of the array is reinforced in a desired direction and suppressed in undesired directions.

An antenna array is a group of multiple active antennas coupled to a common source or load to produce a directive radiation pattern. Usually, the spatial relationship of the individual antennas also contributes to the directivity of the antenna array. Use of the term "active antennas" is intended to describe elements whose energy output is modified due to the presence of a source of energy in the element (other than the mere signal energy which passes through the circuit) or an element in which the energy output from a source of energy is controlled by the signal input. One common application of this is with a standard multiband television antenna, which has multiple elements coupled together. [11]

A microstrip or patch antenna is a low- profile antenna that has a number of advantages over other antennas - - it is lightweight, inexpensive, and easy to integrate with accompanying electronics. While the antenna can be 3- D in structure (wrapped around an object, for example), the elements are usually flat, hence their other name, planar antennas. Note that a planar antenna is not always a patch antenna. [8]

The following drawing shows a patch antenna in its basic form: a flat plate over a ground plane (usually a PC board). The centre conductor of a coax serves as the feed probe to couple electromagnetic energy in and/or out of the patch. The electric field distribution of a rectangular patch excited in its fundamental mode is also indicated.

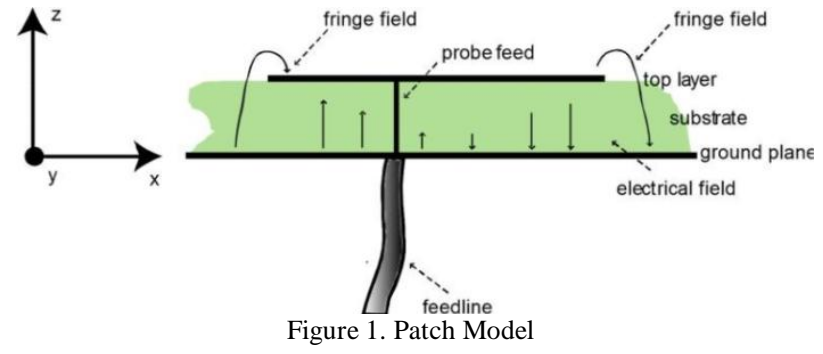

II. LITERATURE REVIEW

The elements of the antenna arrays are rectangular patches. Two feeding mechanisms, aperture coupling and via feed, were implemented and compared. For the RF distribution network and interconnects, a corporate feed approach was used with reactive T-junctions, Wilkinson dividers, or both, for power division. The feed networks were designed using microstrip. The basic multilayer antenna array design consists of 3 layers of cladded laminate material. The metal layers are as follows: 1) patch antennas, 2) ground plane, 3) feed network, and 4) surface-mount components. [1]

This thesis demonstrates several shapes of microstrip array antennas, such as rectangular and triangular patch antennas array. Specifically, $4 \times 1,2 \times 1$, and single element of both shapes are designed and simulated by a full wave simulator (IE3d). Moreover, this paper presents a comparison between both rectangular and triangular antenna arrays. Since, the resonance frequency of these antennas is $2.4 \mathrm{GHz}$, these antennas are suitable for ISM band and WLAN. [2]

The power divider network is designed with $100 \Omega$ characteristic impedance to reduce the effect of power divider network on the radiation pattern. The substrate used is RT Duroid 5880 with $\mathrm{Er}=2.2$ and $\mathrm{h}=20$ mil. The feed network is designed and optimized using ADS 2008A 8446 
$\mathrm{s} / \mathrm{w}$ and then integrated with the array. The patch antenna with inset feed design steps include inset line fed patch parameters calculation using transmission line equations. [3]

\section{III.PROPOSED MODEL}

A microstrip antenna in its simplest configuration consists of a radiating patch on one side of a dielectric substrate, which has a ground plane on the other side. The patch conductors usually made of copper or gold can be virtually assumed to be of any shape. However, conventional shapes are normally used to simplify analysis and performance prediction. The radiating elements and the feed lines are usually photo etched on the dielectric substrate.

\section{A. Design of a Patch Antenna at $2.4 \mathrm{GHZ}$}

Selecting an appropriate substrate of thickness (h) and dielectric constant ( $\mathrm{kr}$ ) for the design of the patch antenna. In present case, we shall use following Dielectric for design:
a.
Height: $1.6 \mathrm{~mm}$
b. Metal Thickness: 1.4 mil (1 oz. Copper i.e.35um)
c. $\quad$ Er: 4.6
d. TanD: 0.001
e. Conductivity: $5.8 \mathrm{E} 7 \mathrm{~S} / \mathrm{m}$

Calculating the physical parameters of the patch antenna as shown in the geometry in Figure 18 using the given formula.

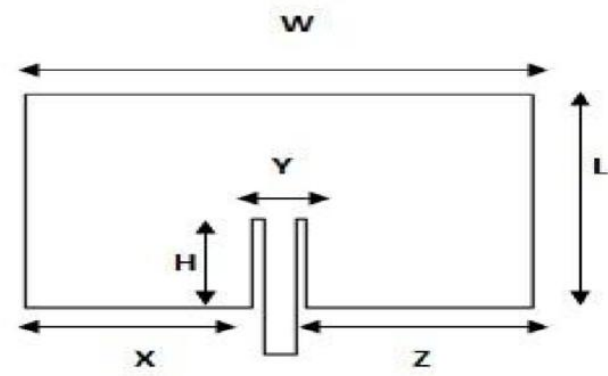

Figure 2. Geometry of the Square Patch Antenna

The depth of the feed line in to the patch is given by,

$$
\mathrm{H}=0.822 * \mathrm{~L} / 2=12 \mathrm{~mm}
$$

The other dimensions are,

$$
\begin{array}{ll}
Y=W / 5 & =5.8 \mathrm{~mm} \\
X=Z=2 W / 5 & =11.7 \mathrm{~mm}
\end{array}
$$

The width and length of the radiating surface is given by,

$$
\mathrm{W}=\mathrm{L}=\frac{\mathrm{c}}{\left(2 \mathrm{f} \sqrt{\varepsilon_{\mathrm{r}}}\right)}=29.2 \mathrm{~mm}
$$

where,

$$
\begin{aligned}
& \text { velocity of light } c=3 \times 10^{8} \mathrm{~m} / \mathrm{s} \\
& \text { Frequency, } f=2.4 \mathrm{GHz} \\
& \text { Relative Permittivity } \varepsilon_{r}=4.6
\end{aligned}
$$

Connect a pin at the feed point of the antenna at the required point and go to the EM setup window and click on Substrate and click on New to accept the 25 mil Alumina template. Define the substrate as below, modify the default substrate height, Er, TanD and conductor height and define it as Copper (select it from Add from Database list). Changing name of the dielectric is optional as it has no bearing on the simulation. Click on cond and check as intrude into the substrate.

Set the Simulation Frequency range as $2.1 \mathrm{GHz}-2.7 \mathrm{GHz}$ (adaptive sweep) and Add a new Single Point of $2.4 \mathrm{GHz}$. Click on Simulate and observe the simulation results in data display as shown below.

\section{B. Design of an Array Antenna}

1. Create the Patch Antenna geometry as per calculations done in 1st lab (or copy/paste)

2. Place Patch Antenna feed point at $(0,0)$ coordinate to make job easier for creation of our patch array.

3. Place a Pin for simulation at the centre of feed line. This step will ensure that all copied instances will also have simulation pins else we will need to keep it manually.

4. Copy/Paste antenna element using Insert$>$ Coordinate Entry and enter required coordinates. Click Apply between every coordinate entry

5. Press Esc button to cancel the command. Select this entire row and then select Insert->Coordinate Entry and enter coordinates.

For Patch Array design, separation between elements are usually kept as $0.7 *$ lambda $-0.8 *$ lambda. In our case $93.75 \mathrm{~mm}$ is $0.75 *$ lambda at $2.4 \mathrm{GHz}$ on $1.6 \mathrm{~mm}$ FR4 substrate.

\section{A. Design}

\section{IV.DESIGN OF LINEAR 1X2 ARRAY}

In order to make fair comparison, the same substrate used in single element $(\mathrm{Er}=4.6$ and thickness $\mathrm{h}=1.6 \mathrm{~mm})$, is used in the $1 \times 2$ array. The configuration of $1 \times 2$ linear rectangular patch antenna array. To obtain $50 \mathrm{Ohms}$ input impedance, feeding line with width $\mathrm{W} 1=4.85 \mathrm{~mm}$ is used. This line is split into two $100 \mathrm{Ohms}$ lines, with width $\mathrm{Wo}_{\mathrm{O}}=1.41 \mathrm{~mm}$. The configuration of $1 \times 2$ linear triangular patch antenna array. The same design procedure, that was used for rectangular, is used in triangular array. It is worth to mention that, the patches (rectangular and triangular) dimensions used in the arrays have same dimensions used in single patches design.

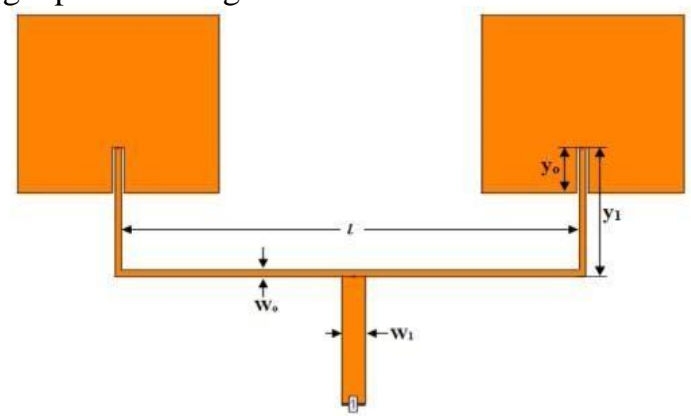

Figure 3. Configuration of $1 \times 2$ linear rectangular patch antenna array www.ijarcce.com 8447 
International Journal of Advanced Research in Computer and Communication Engineering Vol. 3, Issue 11, November 2014

\section{B. Results obtained upon simulations}

For simulation and lab running to obtain the array antenna, Agilent Technologies ADS 2013.06 was used.

After multiple tests running and sampling the results obtained are as shown as below:

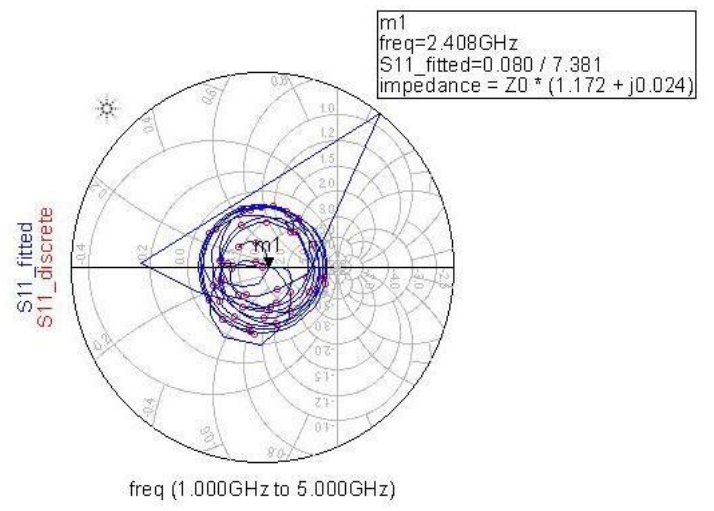

Figure 4. Smith Chart for Impedance Matching

In the figure 5 we can see the results for Return loss of 1x2 Array Antenna. Here we are able to see that spacing between Gain and Directivity decreases as we have placed an antenna array element.

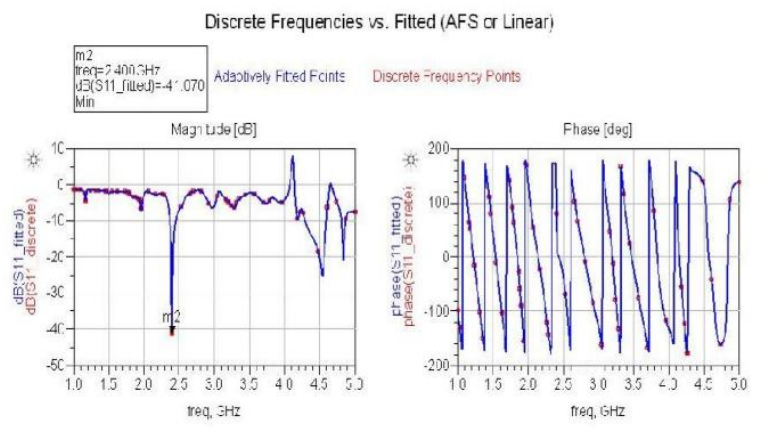

Figure 5. Return loss graph for 1x2 Array Antenna

The figure 6 shows results obtained for 1x2 Linear Array from the simulation. It also mentions the obtained parameter results of it.

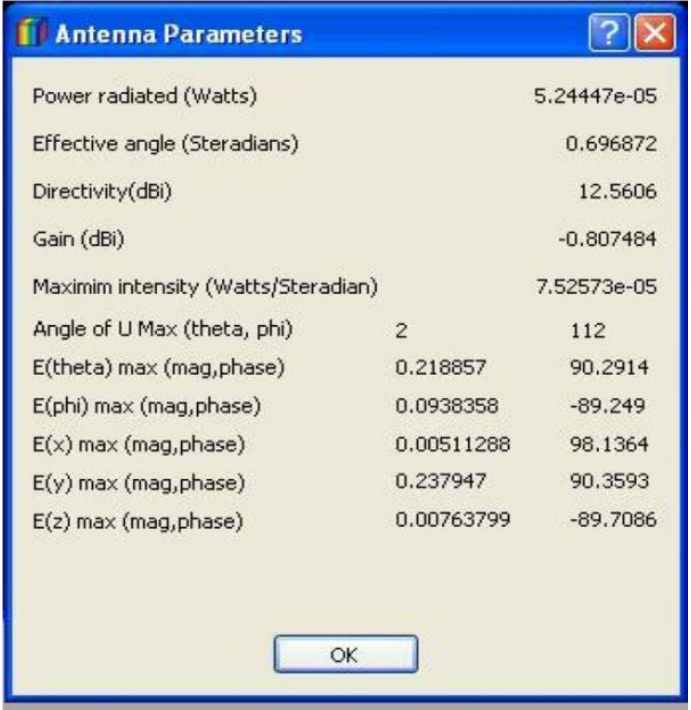

Figure 6. Antenna Parameters

\section{DESIGN OF 2X2 ARRAY ANTENNA}

\section{A. Design}

To design an array we need a feed network which will connect all the four elements. To design the feed network we need to start from the input impedance of the patch. The single element is fed by a $100 \Omega$. So the starting point of the feed will be $100 \Omega$. The scheme of the feed network is as shown in the figure.

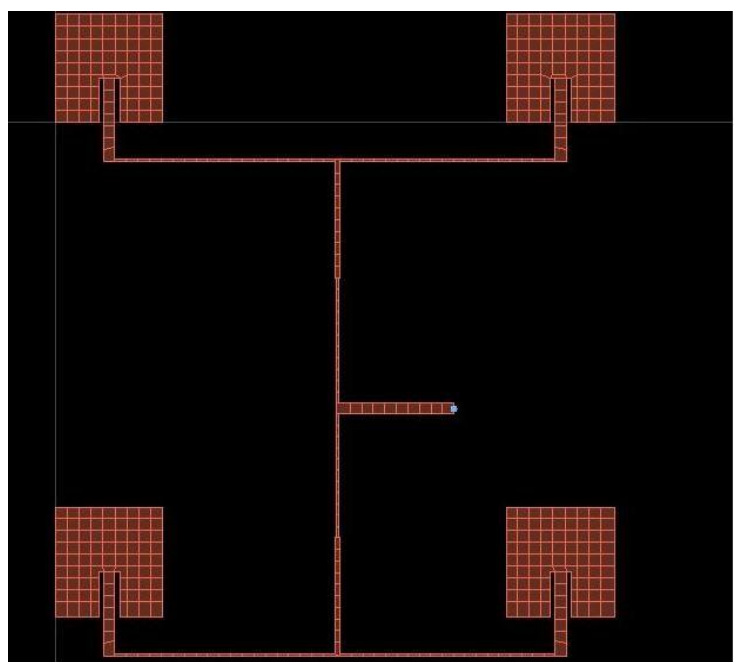

Figure 7. Layout of $2 \times 2$ array antenna

\section{B. Results obtained upon simulations}

In the figure 5 we can see the results for Return loss of $2 \times 2$ Array Antenna. Here we are able to see that spacing between Gain and Directivity still more increases as we have placed one more antenna array element.

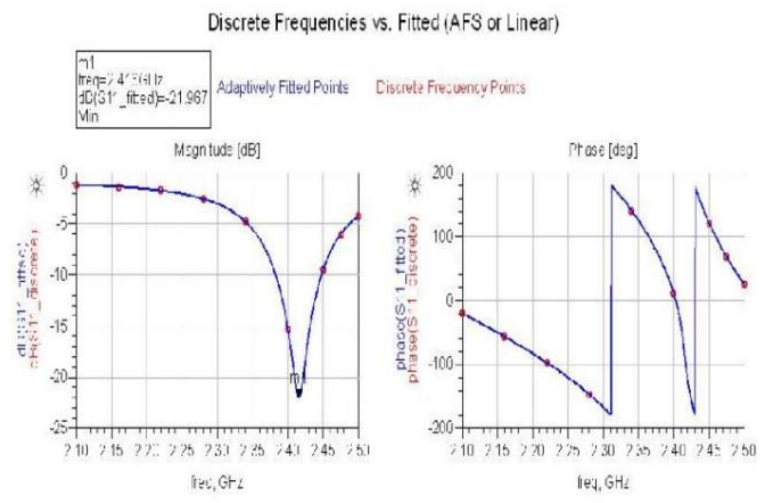

Figure 8. Return loss for 2x2 Array Antenna

The figure 9 shows the smith chart of 2X2 Array Antenna. Here we can see that the HPBW and BWFN decreases, as we have increased the spacing between the antenna elements. 


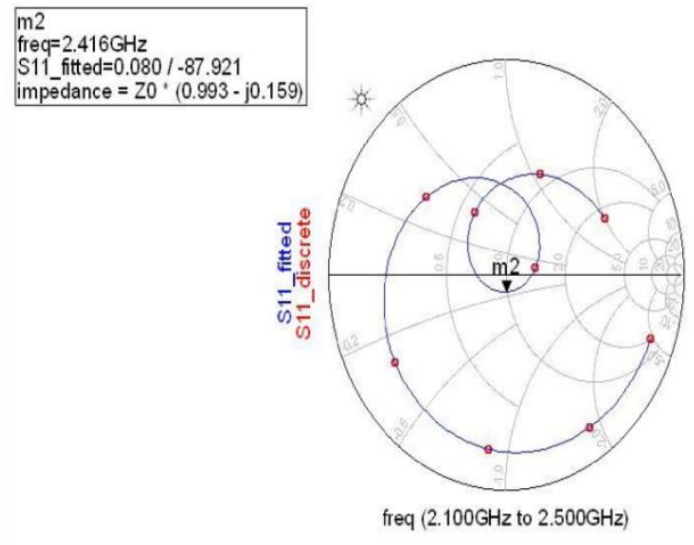

Figure 9. The Smith chart for $2 \times 2$ Array Antenna

The figure below shows the Radiation Pattern of $2 \times 2$ Array antenna. It shows the 3-D side view of Radiation Pattern obtained. From this Radiation Pattern we can see that the beam gets narrower if the number of element in the array increases.

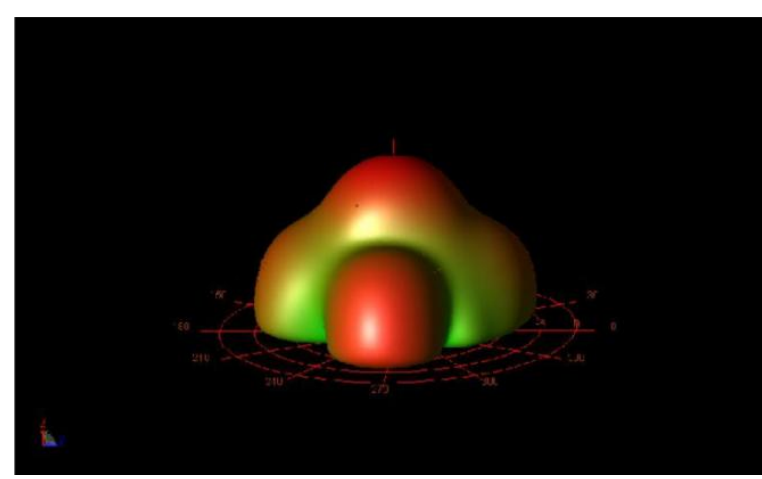

Figure 10. The Radiation Pattern for $2 \times 2$ Array Antenna

\section{VI.CONCLUSION}

In the present work, arrays of Microstrip patch antenna with different types are modeled using finite element method and simulated in ADS 2013. The results are compared with conventional rectangular patch array for $1 \times 4$ and 2x2. By analyzing the results we inferred that

- $\quad$ The HPBW and BWFN decreases as spacing between the array elements increases.

- The gain and directivity increases with the increase in spacing.

- The beam gets narrow if the number of the elements in an array increases.

Considering the area of the element, Rectangular shaped patch gave better gain and directivity at different array models.

Thus, this proposed antenna array is suitable for designing the environmental scanning antenna and Phased array Antenna. The simulation work carried out by us can be made use for the design of any shapes of Microstrip patch antenna.

\section{REFERENCES}

[1] Ana Maria Yepes "Multilayer Antenna Array for Environmental Sensing Application”, 2010.

[2] S. H. Yahya Khraisat, M. Melad Olaimat \& Sharief N. AbdelRazeq, 2011 "Comparison between Rectangular and Triangular Patch Antennas Array"

[3] Network by Amlesh Kumar Singh, Hem Prasad Ghanta, R.K. Samminga and Dr.Anil Kumar Singh "Design of an Inset Fed Microstrip Patch Antenna Array with Integrated Feed”, 2012.

[4] Chandan, B.S.Rai, R.K.Prasad, "Improve Bandwidth by S Shaped Microstrip Patch antenna", International Journal of Emerging Trends in Engineering and Development, ISSN 2249-6149, Issue 3, Vol.1,p.p 641646, January 2013.

[5] Diwakar Singh, Amit Kumar Gupta, R. K. Prasad, "Design and analysis of dual band $\Psi$ - microstrip patch antenna", International Journal of Advances in Engineering and Technology, ISSN: 22311963, Vol. 6, Issue 1, pp. 520-526, March 2013.

[6] Miss Neenansha Jain, Prof. Rajesh Nema, Dr. Anubhuti Khare, Puran Gour, " Dual Band E-shaped Microstrip patch antenna on RT DUROID 5880 Substrate for Pervasive Wireless Communication," International journal of Computer Science and Technologies, ISSN: 0975-9646, Vol.2(3), 2011.

[7] Amit Kumar Gupta, R.K.Prasad and Dr. D.K.Srivastava, "Design and Development of Dual E-shaped Microstrip patch antenna for bandwidth and gain enhancement," IJECET, ISSN: 0976-6464, Volume3, Issue3, OctDec 2012.

[8] www.google.co.in

[9] http://ieeexplore.org

[10] www.zealand.com

[11] www.wikipedia.com 